\title{
The Detection and Clinical Significance of Circulating Tumor Cells in Patients with Renal Cell Carcinoma
}

\author{
Huansheng Wang ${ }^{1,2}$, Benkui Zou ${ }^{2}$, Menglin Bai ${ }^{1 *}$ and Sheng $\mathrm{Li}^{1 *}$ \\ ${ }^{1}$ School of Medicine, Shandong University, China \\ ${ }^{2}$ Department of Urological Surgery, Shandong Cancer Hospital and Institute, Shandong First Medical University and Shandong \\ Academy of Medical Sciences, China
}

*Corresponding author: Sheng Li and Menglin Bai, School of Medicine, Shandong University, China

\section{ARTICLE INFO}

Received: 慧 August 18, 2021

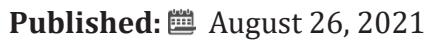

Citation: Huansheng Wang, Benkui Zou, Menglin Bai, Sheng Li. The Detection and Clinical Significance of Circulating Tumor Cells in Patients with Renal Cell Carcinoma. Biomed J Sci \& Tech Res 38(3)2021. BJSTR. MS.ID.006147.

\begin{abstract}
Purpose: The presence of circulating tumor cells (CTC) in patients with renal cell carcinoma (RCC) has potential diagnostic and prognostic significance. We previously applied the CellSearch system (CSS) and the isolation by size of epithelial tumor cells (ISET) method to detect CTCs in RCC patients, and analysis of the 3-year follow-up data indicated that CTC detection correlated significantly with the clinical stage of RCC. The present study aimed to determine the longer-term clinical value of CTC detection for evaluating prognosis in RCC patients.
\end{abstract}

Methods: The present study included 37 RCC patients and 22 healthy individuals who were followed up for 5 years after CTC detection using the CSS and ISET methods. Relevant clinical pathological characteristics were recorded, and patient prognosis was examined by calculating progression-free survival (PFS) and overall survival (OS). Correlations between CTC detection and relevant factors were analyzed.

Results: The CTC detection rates were 18.92\% (7/37) with the CSS and 35.14\% $(13 / 37)$ with ISET in the RCC patients. CTC detection by ISET, but not by the CSS, correlated with clinical tumor node metastasis stage, tumor size and International Metastatic RCC Database Consortium score. However, CTC detection by either method showed no correlation with the PFS or OS of RCC patients.

Conclusion: The CTC detection rate using ISET was higher than that with the CSS. Thus, CTC detection by ISET can serve as a useful supplement to the clinical staging of RCC, although CTC detection by either method did not correlate with the 5-year prognosis of the patients.

Keywords: Renal Cell Carcinoma; Circulating Tumor Cell; CellSearch; Isolation by Size of Epithelial Tumor Cells; Prognosis

\begin{abstract}
Abbreviations: CTC: Circulating Tumor Cells; RCC: Renal Cell Carcinoma; CSS: CellSearch System; ISET: Isolation by Size of Epithelial Tumor Cells; PFS: Progression-Free Survival; OS: Overall Survival; TNM: Tumor Node Metastasis; KPS: Karnofsky Performance Scale; IMDC: International Metastatic RCC Database Consortium; AKP: Alkaline Phosphatase; LDH: Lactate Dehydrogenase; EpCAM: Epithelial Cellular Adhesion Molecule; CTM: Circulating Tumor Microemboli; EMT: Epithelial-to-Mesenchymal Transition; CK: Cytokeratin; MET: Mesenchymal-to-Epithelial Transition
\end{abstract}

\section{Introduction}

Renal cell carcinoma (RCC) arises from the epithelial lining of renal tubules and is the most common renal malignancy, accounting for approximately $85 \%$ of all renal malignancies. It is also the second most common urinary tract tumor after bladder cancer in the field of urology. Although gradual advancements in the early detection rate of RCC have been made, about $30 \%$ of patients have metastatic lesions at the time of diagnosis, and $20 \%-40 \%$ of patients with RCC will experience local recurrence or distant metastasis after treatment [1]. Hematogenous metastasis is a common mechanism of RCC metastasis to distant organs and 
an important cause of the poor prognosis of RCC [2]. Circulating tumor cells (CTCs) are an important medium for the metastasis of RCC [3]. These cells separate from the primary lesion or metastatic lesions and enter the circulatory system via peripheral blood [4]. Early research found that $31 \%$ of metastatic RCC patients with a high baseline level of CTCs will die within 2 years, excluding other factors [5].

With the development of modern molecular biology and medical detection approaches, CTCs are increasingly becoming a hot topic in tumor metastasis research as important biomarkers for cancer diagnosis, treatment response, and postoperative recurrence [6,7]. Specifically, multiple studies have investigated the roles of CTCs in RCC metastasis and prognosis $[3,8]$. CTCs have shown significance in the clinical staging and auxiliary diagnosis of RCC [9]. Studies have also demonstrated a relationship between the CTC detection value and tumor node metastasis (TNM) staging of RCC $[10,11]$. Nayak, et al. suggested that the baseline CTC level in RCC patients is an important predictor of progression-free survival (PFS) [12]. Bluemke, et al. found that the presence of CTCs is significantly correlated with the overall survival (OS) of RCC patients after CTC detection. In their earlier work, Nayak, et al. showed that CTCs are the main culprit for the poor therapeutic effect of targeted tyrosine kinase therapy in patients with metastatic RCC, providing insight for improved drug treatment of renal cancer [6].

Although the detection of CTCs in peripheral blood has been clinically applied in some cancer types, such as breast cancer and prostate cancer, research on detection methods for CTCs in the peripheral blood of RCC patients is relatively scarce, and the success rates of CTC isolation in these patients has been low [1315]. Currently, reported methods for CTC detection in RCC include RT-PCR, the CellSearch system (CSS), immunomagnetic bead sorting (MACS), size-based exclusion, flow cytometry, microfluidic chip capture, and many other methods, and the most commonly used detection methods are isolation by size of epithelial tumor cells (ISET) and the CellSearch system (CSS) $[6,16]$. The CSS is the only FDA-approved method for using CTC detection to monitor treatment efficacy and evaluate prognosis in breast, prostate, colorectal and other cancers in the United States [17]. Allard, et al. first reported that the CSS showed high CTC detection rates in breast cancer, colorectal cancer and other cancers, but the detection rate of CTCs in the peripheral blood of RCC patients was only $25 \%$ [18]. Moreover, in the Han population, the CTC detection rate in the peripheral blood of RCC patients may be as low as $12.5 \%$ [19].

In 2014, our research group reported CTC detection in peripheral blood samples from 40 patients with renal cancer using CTC-biopsy and the Celltracks $\AA$ Autoprep $₫$ automatic detection system [10]. In our later study in 2017, we found that CTC detection by ISET correlated with clinical TNM stage, while CTC detection by CSS did not. Moreover, CTC detection by both approaches showed no correlation with OS or PFS. However, the duration of follow-up was short, and some clinical data were missing. The correlation between CTC detection and the prognosis of patients and its guiding significance for clinical treatment of RCC warrants further investigation. In the present study, CTC detection was performed using the CSS and ISET for 37 patients with renal cancer, who were then followed up for at least 5 years to assess prognosis based on OS and PFS. Here, we present the preliminary findings and discuss the correlation between CTC detection results and the clinical pathological characteristics and prognosis of RCC patients. This study can serve as a reference for the treatment of patients with RCC.

\section{Research Design and Methods}

\section{Study Participants}

From May 2014 to December 2014, a total of 37 patients who were diagnosed with RCC in the Shandong Cancer Hospital were enrolled in this study. The main inclusion criteria were:

1) Age >18 years;

2) Diagnosis of RCC by pathological examination;

3) Initial treatment; and

4) An Eastern Cooperative Oncology Group (ECOG) score $\leq 2$.

Patients with benign renal tumors were excluded from this study. Blood samples were collected from 22 healthy volunteers for a negative control group. For these volunteers, abnormal liver and kidney function was excluded by laboratory examinations, and RCC was excluded by imaging examination. All participants provided informed consent. Patient's baseline characteristics were recorded, including gender, age, Karnofsky Performance Scale (KPS) score, International Metastatic RCC Database Consortium (IMDC) score and the results from hematological examination (platelet count, hemoglobin level, neutrophil count, blood calcium level, alkaline phosphatase [AKP] level, and lactate dehydrogenase [LDH] level) and pathological examination (pathological stage, tumor size, TNM stage, and other clinical pathological factors).

\section{Blood Sample Collection}

Peripheral blood samples were collected following procedures reported previously [10]. Briefly, $14 \mathrm{ml}$ of venous blood was collected into a tube containing an anticoagulant and then divided equally into two parts for CSS and ISET analyses.

\section{CTC Detection Using the CSS}

The CSS is a cell enrichment system based on the expression of the cell surface antigen epithelial cellular adhesion molecule (EpCAM). In the process of detection, CTCs and white blood cells in the blood samples of patients were first enriched using a magnetic fluid containing anti-EpCAM antibody, and then the enriched cells were labeled with CK8/18/19 antibody and CD45 antibody, respectively. DAPI (4',6-diamidino-2-phenylindole) was used for nuclear staining. Finally, the screened EpCAM+/CK+/DAPI+/CD45- 
cells were labeled as CTCs. The CSS analyses were performed by a collaborating research group at Shanghai Dingjing Company, which has testing qualifications granted by Johnson \& Johnson Company (USA). All procedures were completed by trained technicians in strict accordance with the manufacturer's operating standards.

\section{CTC Detection by ISET}

The ISET approach is a method to separate CTCs from peripheral blood based on the physical characteristic of cell size. Our research group has developed a CTC detection system based on ISET technology with independent intellectual property rights through a large number of clinical experiments. Briefly, blood samples are fixed by $0.2 \%$ formaldehyde and then filtered through a porous membrane with a pore size of $8 \mu \mathrm{m}$. The remaining cells are stained with Romanowsky stain. The morphological criteria for CTCs/circulating tumor microemboli (CTM) are as follows:

(i) Irregular chromatin staining;

(ii) Nuclear size $>18 \mu \mathrm{m}$ in diameter;

(iii) Nuclear-cytoplasmic ratio $>0.8$;

(iv) Thickened and wrinkled nuclear membrane; and

(v) Adherence of multiple CTCs to form CTM. Only cells with 4 or more of these features are classified as CTCs/CTM. All images were analyzed and re-analyzed by three experienced pathologists.

\section{Follow-Up}

The 37 RCC patients in this study were followed up every three

\section{Results}

\section{Baseline Characteristics of Patients}

Table 1: Baseline characteristics of patients.

\begin{tabular}{|c|c|}
\hline Characteristics (N=37) & $57.3 \pm 9.13(33-72)$ \\
\hline Age (Years) Median (range) & $29(78.38)$ \\
\hline Sex, $\mathrm{n}(\%)$ & $8(21.62)$ \\
\hline Male & $90.77 \pm 8.39(70-100)$ \\
\hline Female & $245.3 \pm 80.36(114-495)$ \\
\hline KPS Score, Median (range) & $137.2 \pm 18.58(95-168)$ \\
\hline Platelet Count, $\times 10^{9} / \mathrm{L}$, Median (range) & $6.59 \pm 2.43(3.07-10.88)$ \\
\hline Hemoglobin, g/L, Median (range) & $2.74 \pm 0.21(1.89-2.98)$ \\
\hline Neutrophil Count, $\times 10^{9} / \mathrm{L}$, Median (range) & $87.5 \pm 48.90(33-330)$ \\
\hline Ca, mmol/L, median (range) & $156.5 \pm 101.04(98-522)$ \\
\hline AKP, U/L, Median (range) & $16(43.24)$ \\
\hline LDH, U/L, Median (range) & $2(5.41)$ \\
\hline TNM Stage, $\mathrm{n}(\%)$ & $7(18.92)$ \\
\hline I & $12(32.42)$ \\
\hline II & $17(45.95)$ \\
\hline III & \\
\hline IV & \\
\hline T Stage, $\mathrm{n}(\%)$ & \\
\hline T1 & \\
\hline
\end{tabular}

months for at least 5 years after CTC detection. Follow-up included regular re-examination of patients, recurrence and metastasis, and the time of recurrence, time of death, DFS and OS were recorded. The final telephone follow-up was performed in January 2021 or when a patient had died. PFS was defined from the time the patient was tested for CTCs to the time when RCC progression occurred, or the patient died. OS was defined from the time the patient was tested for CTCs until the patient died from any cause.

\section{Statistical Analysis}

SPSS 26.0 software was used for all statistical processing of the experimental data. Average values between the groups with and without CTCs were compared by t test, and rates were compared between the groups by the test or Fisher's exact probability method. Values of $\mathrm{P}<0.05$ indicated statistical significance. Data for continuous variables are presented as mean \pm standard deviation. The $t$ test was used for analysis of continuous data that conformed to normal variance, while the non-parametric test was used for continuous data that did not conform to normal variance. Data for categorical variables are expressed as frequencies (percentages), and differences in these data were tested using the chi-square test or Fisher's exact probability method (when 1/5 of the tables had theoretical frequencies $<5$ or at least 1 theoretical frequency $<1$ ). Survival curves were generated using the Kaplan-Meier method. The log-rank test was used to analyze the survival data and calculate corresponding $\mathrm{P}$ values. Cox proportional risk regression model was used to analyze and evaluate the prognostic factors. $\mathrm{P}<0.05$ indicated that a difference was statistically significant. 


\begin{tabular}{|c|c|}
\hline $\mathrm{T} 2$ & $4(10.81)$ \\
\hline T3 & $15(40.54)$ \\
\hline $\mathrm{T} 4$ & $1(2.70)$ \\
\hline \multicolumn{2}{|c|}{ Tumor Size, n (\%) } \\
\hline$\leq 4 \mathrm{~cm}$ & $10(27.03)$ \\
\hline $4-9.9 \mathrm{~cm}$ & $20(54.05)$ \\
\hline$\geq 10 \mathrm{~cm}$ & $7(18.92)$ \\
\hline \multicolumn{2}{|c|}{ Lymph Node Metastasis, n (\%) } \\
\hline Yes & $4(10.81)$ \\
\hline No & $33(89.19)$ \\
\hline \multicolumn{2}{|c|}{ Distant Metastasis, n (\%) } \\
\hline Yes & $10(27.03)$ \\
\hline No & $27(72.97)$ \\
\hline \multicolumn{2}{|c|}{ IMDC Grade, n (\%) } \\
\hline 1 & $21(56.76)$ \\
\hline 2 & $11(29.73)$ \\
\hline 3 & $4(10.81)$ \\
\hline 4 & $1(2.70)$ \\
\hline $1+2$ & $32(86.49)$ \\
\hline $3+4$ & $5(13.51)$ \\
\hline
\end{tabular}

Note: KPS: Karnofsky Performance Scale; Ca: Calcium; AKP: Alkaline Phosphatase; LDH: Lactate Dehydrogenase; IMDC: International Metastatic RCC Database Consortium.

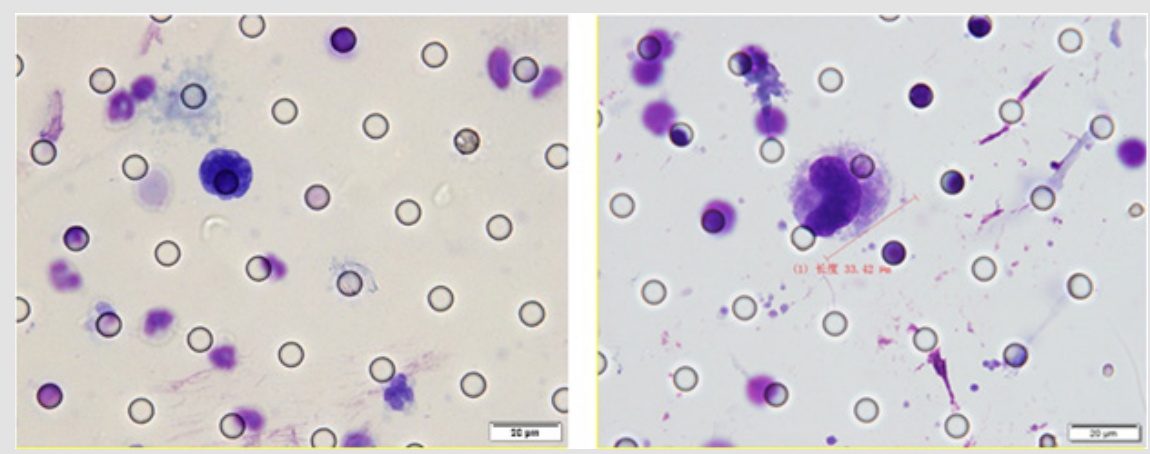

Figure 1: CTC detection by ISET. Representative cellular morphological results for CTCs/CTM detected in the peripheral blood of patients with RCC. Cells isolated by ISET were stained with Romanowsky stain. Cells from healthy controls showed a normal cellular size and morphology (left), and those from RCC patients showed an irregular nuclear shape and abnormally large nuclei (right). Scale bar: $20 \mu \mathrm{m}$.

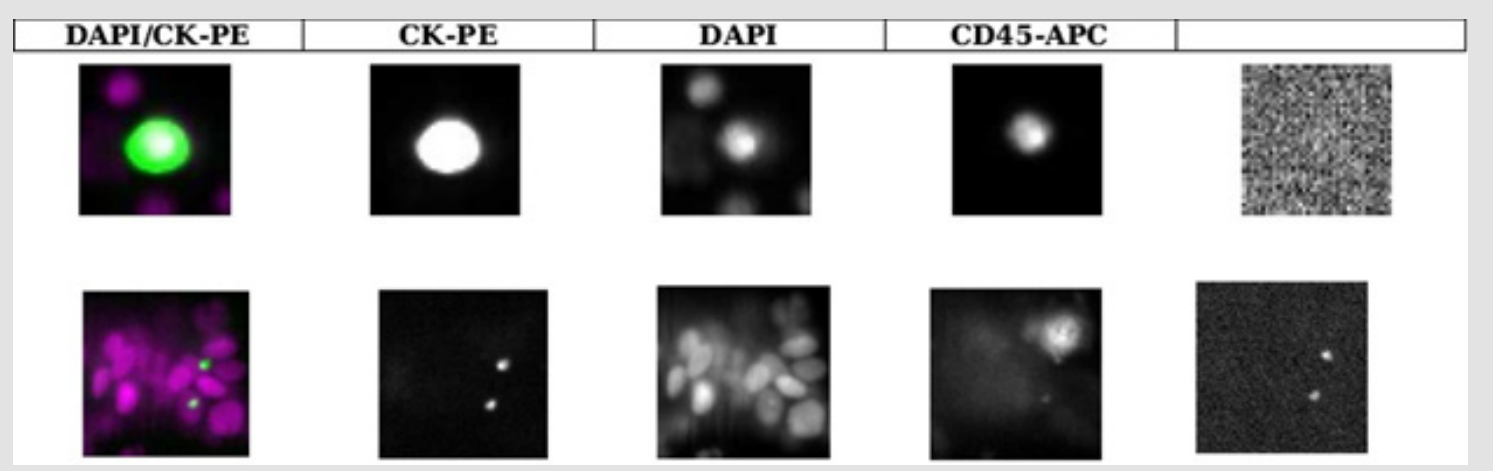

Figure 2: CTC detection results by CSS. Representative cellular staining results of CTCs detected in the peripheral blood of patients with RCC. EpCAM-positive cells were co-stained with fluorescently labeled CK and CD45 antibodies, and nuclei were stained with DAPI. CK+/DAPI+/CD45- cells were considered as CTCs (upper). 
The baseline demographic and clinical characteristics of the 37 included RCC patients are presented in Table 1 . These 37 patients included 34 cases of renal clear cell carcinoma, 2 cases of papillary cell carcinoma, and 1 case of chromophobe cell carcinoma. Notably, the majority of patients were male (78.38\%) and had good KPS scores. Distant metastasis occurred in 10 (27.03\%) patients, and lymph node metastasis in $4(10.81 \%)$ patients. The study population included RCC cases of all TNM stages, T stages, and IMDC grades, as well as tumors distributed across three size ranges $(<4 \mathrm{~cm}, 4-9.9$ $\mathrm{cm}$, and $\geq 10 \mathrm{~cm}$ ).

\section{CTC Detection by ISET Versus CSS}

The CTC detection rates among the 37 patients were $35.14 \%$ (13/37) using the ISET approach (Figure 1) and 18.92\% (7/37) using the CSS (Figure 2). Additionally, CTM were detected only

\section{Correlation between CTC Detection Using the CSS and} Pathologic Factors in RCC Cases

We next investigated correlations between the presence of detectable CTCs and clinical pathological features. By using independent sample t test, test and other methods for data analysis, it was found that CTC detection in the peripheral blood of 37 RCC by ISET and not by the CSS. Statistical analysis using a paired $t$ test showed that the CTC detection rate by ISET was significantly higher than that by the CSS $(\mathrm{P}<0.05)$. However, the chi-square test did not show a significant difference in the detection rates for the two methods ( $\mathrm{P}>0.05)$, but the kappa value of 0.072 indicated poor consistency between the two detection methods (Table 2). No CTCs were detected in peripheral blood samples from healthy controls using either method (Figures $1 \& 2$ ).

Table 2: Comparison of CTC detection by CSS and ISET.

\begin{tabular}{|c|c|c|c|c|c|}
\hline \multirow{2}{*}{ CSS } & \multicolumn{2}{|c|}{ ISET } & \multirow{2}{*}{ Total } & \multirow{2}{*}{ P } & Kappa \\
\cline { 2 - 4 } & Positive & Negative & & & \\
\hline Positive & 3 & 4 & 7 & 0.180 & 0.072 \\
\hline Negative & 10 & 20 & 30 & & \\
\hline Total & 13 & 24 & 37 & & \\
\hline
\end{tabular}

patients using the CSS correlated with none of the following factors: age, gender, KPS score, pre-treatment platelet count, pre-treatment hemoglobin level, pre-treatment neutrophil count, pre-treatment serum calcium level, pre-treatment AKP level, pre-treatment LDH level, pathological stage, TNM stage, tumor size, IMDC score or IMDC classification score (1-2 was classified as low-risk group, 3-4 was classified as high-risk group) (Table 3).

Table 3: Correlation between CTC detection by CSS and pathologic factors in RCC cases.

\begin{tabular}{|c|c|c|c|c|}
\hline & & Presence of CTCs Using CSS & & \\
\hline & $\mathrm{N}=37$ & Positive & Negative & $\mathbf{P}$ \\
\hline Age (Years), Median \pm SD & 37 & $61.57 \pm 7.68$ & $55.30 \pm 8.91$ & 0.095 \\
\hline Sex, n (\%) & & & & 1.000 \\
\hline Male & $29(78.38)$ & $6(16.22)$ & $23(62.16)$ & \\
\hline Female & $8(21.62)$ & $1(2.70)$ & $7(18.92)$ & \\
\hline ECOG, n (\%) & & & & 1.000 \\
\hline 0 & $35(94.60)$ & $7(18.92)$ & $28(75.68)$ & \\
\hline 1 & $1(2.70)$ & $0(0.00)$ & $1(2.70)$ & \\
\hline 2 & $1(2.70)$ & $0(0.00)$ & $1(2.70)$ & \\
\hline Platelet Count, $\times 10^{9} / \mathrm{L}$, Median \pm SD & & $269.86 \pm 61.04$ & $256.00 \pm 87.74$ & 0.696 \\
\hline Hemoglobin, g/L, Median \pm SD & & $132.57 \pm 15.164$ & $136.97 \pm 19.60$ & 0.583 \\
\hline Neutrophil Count, $\times 10^{9} / \mathrm{L}$, Median \pm SD & & $5.01 \pm 1.83$ & $4.15 \pm 1.69$ & 0.242 \\
\hline Serum Calcium, mmol/L, Median \pm SD & & $2.40 \pm 0.169$ & $2.36 \pm 0.178$ & 0.561 \\
\hline AKP, U/L, Median \pm SD & & $90.57 \pm 39.71$ & $106.33 \pm 61.76$ & 0.526 \\
\hline LDH, U/L, Median \pm SD & & $241.86 \pm 177.03$ & $172.07 \pm 73.71$ & 0.326 \\
\hline \multicolumn{5}{|l|}{ TNM Stage, $\mathrm{n}(\%)$} \\
\hline I & $16(43.24)$ & $1(2.70)$ & $15(40.54)$ & 0.164 \\
\hline II & $2(5.40)$ & $1(2.70)$ & $1(2.70)$ & \\
\hline III & 7 (18.92) & $1(2.70)$ & $6(16.22)$ & \\
\hline IV & $12(32.43)$ & $4(10.81)$ & $8(21.62)$ & \\
\hline Total & 37 (100.00) & 7 (18.92) & 30 (81.08) & \\
\hline I-II & $18(48.65)$ & $2(5.40)$ & $16(43.24)$ & 0.405 \\
\hline III-IV & $19(51.35)$ & $5(13.51)$ & $14(37.84)$ & \\
\hline T Stage, n (\%) & & & & 0.217 \\
\hline $\mathrm{T} 1$ & 17 (45.95) & $2(5.40)$ & $15(40.54)$ & \\
\hline $\mathrm{T} 2$ & $4(10.81)$ & $1(2.70)$ & $3(8.11)$ & \\
\hline
\end{tabular}




\begin{tabular}{|c|c|c|c|c|}
\hline T3 & $15(40.54)$ & $3(8.11)$ & $12(32.43)$ & \\
\hline $\mathrm{T} 4$ & $1(2.70)$ & $1(2.70)$ & $0(0.00)$ & \\
\hline Lymph Node Metastasis, n (\%) & & & & 1.000 \\
\hline No & $33(89.19)$ & $6(16.22)$ & 27 (72.97) & \\
\hline Yes & $4(10.81)$ & $1(2.70)$ & $3(8.11)$ & \\
\hline Distant Metastasis, n (\%) & & & & 0.360 \\
\hline No & 27 (72.97) & $4(10.81)$ & $23(94.60)$ & \\
\hline Yes & $10(27.03)$ & $3(8.11)$ & $7(18.92)$ & \\
\hline Tumor Size, n (\%) & & & & 0.852 \\
\hline$\leq 4 \mathrm{~cm}$ & $10(27.03)$ & $1(2.70)$ & $9(24.32)$ & \\
\hline $4-9.9 \mathrm{~cm}$ & $20(54.05)$ & $5(13.51)$ & $15(40.54)$ & \\
\hline$\geq 10 \mathrm{~cm}$ & 7 (16.22) & $1(2.70)$ & $6(18.92)$ & \\
\hline \multicolumn{5}{|l|}{ IMDC Score, n (\%) } \\
\hline 1 & $21(56.76)$ & $3(8.11)$ & $18(48.65)$ & 0.581 \\
\hline 2 & $11(29.73)$ & $3(8.11)$ & $8(21.62)$ & \\
\hline 3 & $4(10.81)$ & $1(2.70)$ & $3(8.11)$ & \\
\hline 4 & $1(2.70)$ & $0(0.00)$ & $1(2.70)$ & \\
\hline Total & $37(100.00)$ & 7 (18.92) & $30(81.08)$ & \\
\hline $1-2$ & $32(86.49)$ & $6(16.22)$ & $26(70.27)$ & 1.000 \\
\hline $3-4$ & $5(13.51)$ & $1(2.70)$ & $4(10.81)$ & \\
\hline
\end{tabular}

Correlation between CTC/CTM Detection by ISET and detection by ISET correlated significantly with the pathological Pathologic Factors in RCC Cases stage, tumor size and IMDC classification score of the patients (all $\mathrm{P}<0.05$ ) but showed no correlation with other pathologic factors

Among the 37 RCC patients in this study, CTCs were detected by ISET in 13 patients, and CTM in 4 patients. Data analysis using independent sample t-test, test and other methods found that CTC (all P>0.05; Table 4). CTM detection by ISET correlated only with the baseline LDH level $(\mathrm{P}<0.05$; Table 5).

Table 4: Correlation between CTC detection by ISET and pathologic factors in RCC cases.

\begin{tabular}{|c|c|c|c|c|}
\hline & & Presence of CTCs by ISET & & \\
\hline & $\mathrm{N}=37$ & Positive & Negative & $\mathbf{P}$ \\
\hline Age (Years), Median \pm SD & 37 & $56.69 \pm 10.086$ & $56.38 \pm 8.495$ & 0.920 \\
\hline Sex, n (\%) & & & & 0.413 \\
\hline Male & $29(78.38)$ & $9(24.32)$ & $20(54.05)$ & \\
\hline Female & $8(21.62)$ & $4(10.81)$ & $4(10.81)$ & \\
\hline ECOG n (\%) & & & & 0.586 \\
\hline 0 & $35(94.60)$ & $12(32.43)$ & $23(62.16)$ & \\
\hline 1 & $1(2.70)$ & $1(2.70)$ & $0(0.00)$ & \\
\hline 2 & $1(2.70)$ & $0(0.00)$ & $1(2.70)$ & \\
\hline Platelet Count, ×109/L, Median \pm SD & & $256.2 \pm 92.0$ & $262.5 \pm 80.9$ & 0.832 \\
\hline Hemoglobin, g/L, Median \pm SD & & $130.92 \pm 21.411$ & $138.96 \pm 16.918$ & 0.218 \\
\hline $\begin{array}{c}\text { Neutrophil Count, } \times 109 / \mathrm{L} \text {, } \\
\text { Median } \pm \text { SD }\end{array}$ & & $4.01 \pm 1.58$ & $4.48 \pm 1.81$ & 0.432 \\
\hline $\begin{array}{l}\text { Serum Calcium, mmol/L, } \\
\text { Median } \pm \text { SD }\end{array}$ & & $2.4077 \pm 0.2263$ & $2.3458 \pm 0.14$ & 0.311 \\
\hline AKP, U/L, Median \pm SD & & $122.23 \pm 70.53$ & $93.125 \pm 48.74$ & 0.148 \\
\hline LDH, U/L, Median \pm SD & & $207.92 \pm 133.70$ & $173.00 \pm 80.34$ & 0.326 \\
\hline \multicolumn{5}{|l|}{ TNM Stage, n (\%) } \\
\hline I & $16(43.24)$ & $2(5.40)$ & $14(37.84)$ & 0.022 \\
\hline II & $2(5.40)$ & $0(0.00)$ & $2(5.40)$ & \\
\hline III & 7 (18.92) & $4(10.81)$ & 3 (8.10) & \\
\hline
\end{tabular}




\begin{tabular}{|c|c|c|c|c|}
\hline IV & $12(32.43)$ & 7 (18.92) & $5(13.51)$ & \\
\hline Total & $37(100.00)$ & $13(35.14)$ & $24(64.87)$ & \\
\hline I-II & $18(48.65)$ & $2(5.40)$ & $16(43.24)$ & 0.005 \\
\hline III-IV & $19(51.35)$ & $11(29.73)$ & $8(21.62)$ & \\
\hline T Stage, n (\%) & & $13(35.14)$ & $24(64.87)$ & 0.093 \\
\hline $\mathrm{T} 1$ & $17(45.95)$ & $3(8.10)$ & $14(37.84)$ & \\
\hline $\mathrm{T} 2$ & $4(10.81)$ & $2(5.40)$ & $2(5.40)$ & \\
\hline T3 & $15(40,54)$ & 7 (18.92) & $8(21.62)$ & \\
\hline $\mathrm{T} 4$ & $1(2.70)$ & $1(2.70)$ & $0(0.00)$ & \\
\hline Lymph Node Metastasis, n (\%) & & & & 0.140 \\
\hline No & $33(89.19)$ & $13(35.14)$ & $19(51.35)$ & \\
\hline Yes & $4(10.81)$ & $0(0.00)$ & $5(13.51)$ & \\
\hline Distant Metastasis, n (\%) & & & & 0.118 \\
\hline No & 27 (72.97) & $7(18.92)$ & $20(54.05)$ & \\
\hline Yes & $10(27.02)$ & $6(16.22)$ & $4(10.81)$ & \\
\hline Tumor Size, n (\%) & & & & 0.012 \\
\hline$\leq 4 \mathrm{~cm}$ & $10(27.02)$ & $0(0.00)$ & $10(27.02)$ & \\
\hline $4-9.9 \mathrm{~cm}$ & $20(54.05)$ & $9(24.32)$ & $11(29.73)$ & \\
\hline$\geq 10 \mathrm{~cm}$ & 7 (18.92) & $4(10.81)$ & $3(8.10)$ & \\
\hline \multicolumn{5}{|l|}{ IMDC Score, n (\%) } \\
\hline 1 & $21(56.76)$ & 7 (18.92) & $14(37.84)$ & 0.096 \\
\hline 2 & $11(29.73)$ & $2(5.40)$ & $9(24.32)$ & \\
\hline 3 & $4(10.81)$ & $3(8.10)$ & $1(2.70)$ & \\
\hline 4 & $1(2.70)$ & $1(2.70)$ & $0(0.00)$ & \\
\hline Total & $37(100.00)$ & $13(35.13)$ & $24(64.86)$ & \\
\hline $1-2$ & 32 (86.49) & $9(24.32)$ & $23(62.16)$ & 0.042 \\
\hline $3-4$ & $5(13.51)$ & $4(10.81)$ & $1(2.70)$ & \\
\hline
\end{tabular}

Table 5: Correlation between CTM detection by ISET and pathologic factors in RCC cases.

\begin{tabular}{|c|c|c|c|c|}
\hline & & Presence of CTM by ISET & & \\
\hline & $\mathrm{N}=37$ & Positive & Negative & $\mathbf{P}$ \\
\hline Age (Years), Median \pm SD & 37 & $50.50 \pm 12.34$ & $57.21 \pm 8.41$ & 0.159 \\
\hline Sex, n (\%) & & & & 0.198 \\
\hline Male & $29(78.38)$ & $2(5.40)$ & $27(72.97)$ & \\
\hline Female & $8(21.62)$ & $2(5.40)$ & $6(16.21)$ & \\
\hline ECOG, n (\%) & & & & 1.000 \\
\hline 0 & $35(94.59)$ & $4(10.81)$ & $31(83.78)$ & \\
\hline 1 & $1(2.70)$ & $0(0.00)$ & $1(2.70)$ & \\
\hline 2 & $1(2.70)$ & $0(0.00)$ & $1(2.70)$ & \\
\hline Platelet Count, $\times 10^{9} / \mathrm{L}$, Median \pm SD & & $244.75 \pm 59.23$ & $260.30 \pm 85.754$ & 0.728 \\
\hline Hemoglobin, g/L, Median \pm SD & & $134.25 \pm 18.118$ & $136.36 \pm 19.062$ & 0.835 \\
\hline Neutrophil Count, $\times 109 / \mathrm{L}$, Median \pm SD & & $3.073 \pm 0.700$ & $4.467 \pm 1.756$ & 0.129 \\
\hline Serum Calcium, mmol/L, Median \pm SD & & $2.385 \pm 0.113$ & $2.365 \pm 0.182$ & 0.836 \\
\hline $\mathrm{AKP}, \mathrm{U} / \mathrm{L}, \mathrm{Median} \pm \mathrm{SD}$ & & $99.75 \pm 25.42$ & $103.79 \pm 61.10$ & 0.898 \\
\hline $\mathrm{LDH}, \mathrm{U} / \mathrm{L}, \mathrm{Median} \pm \mathrm{SD}$ & & $280.25 \pm 159.84$ & $173.76 \pm 89.47$ & 0.047 \\
\hline \multicolumn{5}{|l|}{ TNM Stage, n (\%) } \\
\hline I & $16(43.24)$ & $1(2.70)$ & $15(40.54)$ & 0.727 \\
\hline II & $2(5.40)$ & $0(0.00)$ & $2(5.40)$ & \\
\hline III & $7(18.91)$ & $1(2.70)$ & $6(16.21)$ & \\
\hline IV & $12(32.43)$ & $2(5.40)$ & $10(27.02)$ & \\
\hline
\end{tabular}




\begin{tabular}{|c|c|c|c|c|}
\hline Total & 37() & $13(35.14)$ & $24(64.86)$ & \\
\hline I-II & $18(48.65)$ & $1(2.70)$ & $17(45.95)$ & 0.604 \\
\hline III-IV & $19(51.35)$ & $3(8.10)$ & $16(43.24)$ & \\
\hline T Stage, n (\%) & & $4(10.81)$ & 33 (89.19) & 0.389 \\
\hline $\mathrm{T} 1$ & $17(45.95)$ & $1(2.70)$ & $16(43.24)$ & \\
\hline $\mathrm{T} 2$ & $4(10.81)$ & $1(2.70)$ & $3(8.10)$ & \\
\hline T3 & $15(40.54)$ & $2(5.40)$ & $13(35.14)$ & \\
\hline $\mathrm{T} 4$ & $1(2.70)$ & $0(0.00)$ & $1(2.70)$ & \\
\hline Lymph Node Metastasis, n (\%) & & & & $1.000 \mathrm{c}$ \\
\hline No & $33(89.19)$ & $4(10.81)$ & $29(78.38)$ & \\
\hline Yes & $4(10.81)$ & $0(0.00)$ & $4(10.81)$ & \\
\hline Distant Metastasis, n (\%) & & & & 0.291 \\
\hline No & $27(72.97)$ & $2(5.40)$ & $25(67.57)$ & \\
\hline Yes & $10(27.02)$ & $2(5.40)$ & $8(21.62)$ & \\
\hline Tumor Size, n (\%) & & & & 0.497 \\
\hline$<4 \mathrm{~cm}$ & $10(27.02)$ & $0(0.00)$ & $10(27.02)$ & \\
\hline $4-9.9 \mathrm{~cm}$ & $20(54.05)$ & $3(8.10)$ & $17(4.95)$ & \\
\hline$\geq 10 \mathrm{~cm}$ & 7 (18.92) & $1(2.70)$ & $6(16.21)$ & \\
\hline IMDC Score & & $3(8.10)$ & $18(48.65)$ & 0.373 \\
\hline 1 & $21(56.76)$ & $0(0.00)$ & $11(29.73)$ & \\
\hline 2 & $11(29.73)$ & $1(2.70)$ & $3(8.10)$ & \\
\hline 3 & $4(10.81)$ & $0(0.00)$ & $1(2.70)$ & \\
\hline 4 & $1(2.70)$ & 13 (35.13) & $24(64.86)$ & \\
\hline Total & $37(100.00)$ & $3(8.10)$ & $29(78.38)$ & 0.456 \\
\hline $1-2$ & $32(86.49)$ & $1(2.70)$ & $4(10.81)$ & \\
\hline $3-4$ & $5(13.51)$ & $3(9.10)$ & $18(48.65)$ & 0.373 \\
\hline
\end{tabular}

\section{Correlation between CTC Detection and Prognosis of RCC Patients}

Table 6: Correlation between CTC detection and RCC prognosis.

\begin{tabular}{|c|c|c|c|}
\hline Factors & HR & $95 \% \mathrm{CI}$ & $\mathbf{P}$ \\
\hline \multicolumn{4}{|c|}{ OS } \\
\hline \multicolumn{4}{|c|}{ Presence of CTCs (by CSS) } \\
\hline$\geq 1$ & 1.187 & $0.326-4.316$ & 0.795 \\
\hline$<1$ & 1 & & \\
\hline \multicolumn{4}{|c|}{ Presence of CTCs (by ISET) } \\
\hline$\geq 1$ & 1.45 & $0.473-4.442$ & 0.516 \\
\hline$<1$ & 1 & & \\
\hline \multicolumn{4}{|c|}{ Presence of CTM (by ISET) } \\
\hline$\geq 1$ & 0.76 & $0.099-5.848$ & 0.792 \\
\hline$<1$ & 1 & & \\
\hline \multicolumn{4}{|c|}{ PFS } \\
\hline \multicolumn{4}{|c|}{ Presence of CTCs (by CSS) } \\
\hline$\geq 1$ & 1.031 & $0.287-3.698$ & 0.963 \\
\hline$<1$ & 1 & & \\
\hline \multicolumn{4}{|c|}{ Presence of CTCs (by ISET) } \\
\hline$\geq 1$ & 2.012 & $0.695-5.822$ & 0.197 \\
\hline$<1$ & 1 & & \\
\hline \multicolumn{4}{|c|}{ Presence of CTM (by ISET) } \\
\hline$\geq 1$ & 0.741 & $0.097-5.670$ & 0.773 \\
\hline$<1$ & 1 & & \\
\hline
\end{tabular}


A total of five patients were lost to follow-up in 5 years, for a loss to follow-up rate of $13.51 \%$ (5/37). For the 32 RCC patients included in the final survival analyses, Cox regression analysis and univariate analysis showed no correlation between CTC detection by either method and the 5-year PFS or OS (P>0.05; Table 6).

\section{Correlation between Therapy Methods and Prognosis of RCC Patients}

The 32 patients included in the final survival analyses were treated using the following methods: surgery + targeted therapy in 10 patients $(25.00 \%, 8 / 32)$, surgery + interleukin- 2 in 24 patients $(65.63 \%, 21 / 32)$, surgery + observation in 1 patient $(3.13 \%, 1 / 32)$, transcatheter arterial embolization in 1 patient $(3.13 \%, 1 / 32)$, and Traditional Chinese Medicine in 1 patient (3.13\%, 1/32). Univariate analysis of treatment modality and prognostic indicators (5-year PFS and 5-year OS) demonstrated a high correlation between treatment modality and patient prognosis $(\mathrm{P}<0.05$; Table 7$)$.

Table 7: Correlation between therapy methods and prognostic indicators.

\begin{tabular}{|c|c|c|c|c|c|c|}
\hline Independent Factors & \multicolumn{3}{|c|}{ Univariate Analysis } & \multicolumn{3}{c|}{ Multivariate Analysis } \\
\hline & HR & $\mathbf{9 5 \%}$ CI & P & HR & $\mathbf{9 5 \%}$ CI & P \\
\hline CTCs (ISET) (Positive/Negative) & 2.012 & $0.695-5.822$ & 0.197 & 2.419 & $0.280-20.880$ & 0.422 \\
\hline CTCs (CSS) (Positive/Negative) & 1.031 & $0.287-3.698$ & 0.963 & 0.332 & $0.065-1.707$ & 0.187 \\
\hline CTM (ISET) (Positive/Negative) & 0.741 & $0.097-5.670$ & 0.773 & 0.943 & $0.117-7.579$ & 0.956 \\
\hline \multicolumn{7}{|c|}{ Therapy Method } \\
\hline $\begin{array}{c}\text { (Operative and Targeted Therapy/ } \\
\text { Operative and Immunotherapy) }\end{array}$ & 0.038 & $0.008-0.181$ & 0 & & \\
\hline
\end{tabular}

Table 8: Correlation between CTC detection and prognosis.

\begin{tabular}{|c|c|c|c|c|c|c|}
\hline \multirow{2}{*}{ Independent Factors } & \multicolumn{3}{|c|}{ Univariate Analysis } & \multicolumn{3}{|c|}{ Multivariate Analysis } \\
\hline & HR & $95 \% \mathrm{CI}$ & $\mathbf{P}$ & HR & $95 \% \mathrm{CI}$ & $\mathbf{P}$ \\
\hline CTCs (ISET) (Positive/Negative) & 1.45 & $0.473-4.442$ & 0.516 & 2.35 & $0.271-20,363$ & 0.438 \\
\hline CTCs (CSS) (Positive/Negative) & 1.187 & $0.326-4.316$ & 0.795 & 0.228 & $0.040-1.306$ & 0.097 \\
\hline CTM (ISET) (Positive/Negative) & 0.76 & $0.099-5.848$ & 0.792 & 1.294 & $0.162-10.351$ & 0.808 \\
\hline \multicolumn{7}{|c|}{ Therapy Method } \\
\hline $\begin{array}{l}\text { (Operative and Targeted Therapy/ } \\
\text { Operative and Immunotherapy) }\end{array}$ & 0.02 & $0.002-0.164$ & 0 & & & \\
\hline
\end{tabular}

\section{Correlation of CTC Detection by ISET or with the CSS with} Therapy Methods and Prognosis of RCC Patients

Finally, dual-factor and Cox analysis of the correlation of CTC/ CTM detection by ISET or CSS with the treatment methods and 5 -year PFS/OS suggested found no significant correlation $(\mathrm{P}>0.05$; Table 8).

\section{Discussion}

Hematogenous metastasis is the main route of RCC metastasis, and studies have shown that CTCs play an important role in the process of tumor metastasis [20,21]. At present, CTCs are used as a form of "liquid biopsy" for assessment of the risks of tumor metastasis and recurrence, for real-time evaluation of the response to therapy, for understanding the blocking mechanism of tumor targeted therapy, and for monitoring cancer progression [22], but the specific role of CTCs in the process of tumor metastasis remains to be further elucidated.

In 2014, our research group performed hematology examination of blood samples from 22 healthy volunteers who were confirmed to be tumor-free and searched for CTCs in the peripheral blood by ISET and using the CSS. No tumor cells were found in the blood of healthy individuals. In the same year, we applied the same techniques to detect CTCs in peripheral blood samples from 37 patients with RCC. Peripheral blood samples from each patient were used for CTC detection by both ISET and the CSS. We found that the CTC/CTM detection rate with the ISET approach was $35.14 \%$ (13/37), which was higher than that achieved with the CSS $(18.92 \%, 7 / 37 ; \mathrm{P}<0.05)$. Moreover, the ISET method could detect CTM, whereas the CSS method could not. We believe that these differences in detection may be attributable to differences in the detection principles between the two technologies. Epithelialto-mesenchymal transition (EMT) is a biological process in which epithelial cells transform into cells with a mesenchymal phenotype through a specific process [23].

Research has suggested that tumor cells derived from epithelial cells must undergo EMT to acquire the ability to detach from the primary tumor, enter the blood circulation, detach from blood vessels, and form new secondary foci [24]. Cells that have undergone EMT show down-regulated expression of epithelial genes, such as EpCAM, E-cadherin and cytokeratin (CK) and exhibit reduced integral protein-mediated adhesion to extracellular matrix, facilitating their entry into the blood circulation [25]. In addition, EMT can lead to increased expression of interstitial genes, such as elastin and matrix metalloproteinases, thereby promoting the dissolution and destruction of extracellular matrix 
and promoting cell metastasis [26]. However, tumor cells have been shown to break through the vascular basement membrane and colonize locally to form new metastatic tumors, after which they undergo mesenchymal-to-epithelial transition (MET) to regain the characteristics of epithelial cells and proliferate to form tumor metastases [27].

The CSS detection system is a cell enrichment technology based on the cell markers EpCAM and CK, which may explain the relatively low CTC detection rate for this method in patients with RCC. In 2013, Zhang, et al. used the CSS to detect CTCs in peripheral blood samples from eight patients with renal cancer, and no CTCs were found. However, in 2011, Gradilone, et al. used the same system to detect CTCs in peripheral blood of 25 patients with renal cancer and found CTCs in samples from four patients $(16 \%, 4 / 25)$ [28]. In summary, we believe that the low EpCAM and CK expression levels on CTCs in the peripheral blood of RCC patients contributed to the low detection rate achieved with the CSS detection system in renal cancer, resulting in the large number of false negative results. We propose that the detection rate with the ISET method was significantly higher than that with the CSS, because the ISET detection process does not depend on the expression of cell surface markers and is not significantly affected by EMT.

CTM form via a type of tumor cell adhesion among CTCs that occurs via cadherin-mediated tumor adhesion molecules and the junction proteins secreted by tumor cells. CTM is more invasive than CTCs [28], and the high invasivity of CTM is attributed to the complex cellular composition of CTM, which includes platelets, immune cells, fibroblasts, and other cell types in addition to CTCs [29]. Previous research showed that CTM in the peripheral blood of tumor patients undergo extensive EMT [30]. Platelets mediate the adhesion of CTM to the vascular endothelium and promote its extravasation. Platelets also can simultaneously release transforming growth factor $\beta$ (TGF- $\beta$ ) and induce EMT of CTM [31]. Notably, detection techniques based partly on the expression of epithelial markers, such as the CSS, cannot detect CTM in the peripheral blood of tumor patients. In the present study, CTM were found in the peripheral blood of four RCC patients using the ISET approach, but the CSS failed to detect CTM in the peripheral blood in RRC patients, which was consistent with the results of previous studies.

In summary, we believe that the differing detection rates between the two technologies is mainly related to their detection principles. The ISET technique isolates CTCs according to cell size, and thus, the results are not influenced by changes in the expression of epithelial markers. However, the CSS cannot detect CTCs or CTM with EMT changes, while the ISET technology remains feasible. Further research is needed to investigate additional reasons for the low CTC detection rate in the peripheral blood of RCC patients. This study showed that CTCs and CTM detection by ISET as well as using CSS technology had no correlation with the 5-year PFS and OS of RCC patients. Considering the small sample size of this study and the high rate of loss to follow-up (13.51\%), future studies with a larger sample size are needed to confirm the benefits of CTC detection in RCC.

\section{Acknowledgement}

This study was supported by the project of major innovation and technology sponsored by Department of Science and Technology of Shandong Province (Grant Number: 2017CXGC1204). Sincerely acknowledgements to Dr. Zuowei Lu and Dr. Min Liu for assistance with cytopathologic analysis. The authors would also like to thank Wuhan YZY Medical Technological Co. Ltd. and United States Johnson \& Johnson Medical Companies for their outstanding technical support.

\section{Authors' Contributions}

Design and final approval of the manuscript: XXX. Clinical data collection: XXX

\section{Funding}

This study was supported by the Jinan university funding projects (2019GXRC020).

\section{Declaration}

\section{Conflict of Interest}

The authors declare that there is no conflict of interest regarding the publication of this article.

\section{Ethics Approval}

The experimental protocol was established, according to the ethical guidelines of the Helsinki Declaration and was approved by ethics Committee of Shandong Cancer Hospital.

\section{Informed Consent}

All participants provided informed consent.

\section{References}

1. Lam JS, Shvarts O, Leppert JT, Figlin RA, Belldegrun AS (2005) Renal cell carcinoma 2005: new frontiers in staging, prognostication and targeted molecular therapy. J Urol 173(6): 1853-1862.

2. Meacci E, Nachira D, Zanfrini E, Evangelista J, Triumbari EKA, et al. (2021) Prognostic Factors Affecting Survival after Pulmonary Resection of Metastatic Renal Cell Carcinoma: A Multicenter Experience. Cancers (Basel) 13(13): 3258

3. Zhang X, Guo X, Zong Y, Xu C, Wang J, et al. (2021) CTCs detection from intraoperative salvaged blood in RCC-IVC thrombus patients by negative enrichment and iFISH identification: a preliminary study. BMC Urol 21: 89.

4. Bidard FC, Ferrand FR, Huguet F, Hammel P, Louvet C, et al. (2012) Disseminated and circulating tumor cells in gastrointestinal oncology. Crit Rev Oncol Hematol 82(2): 103-115.

5. Blümke K, Bilkenroth U, Schmidt U, Melchior A, Füssel S, et al. (2005) Detection of circulating tumor cells from renal carcinoma patients: ex- 
periences of a two-center study. Oncol Rep 14(4): 895-899.

6. Palmela Leitão T, Miranda M, Polido J, Morais J, Corredeira P, et al. (2021) Circulating tumor cell detection methods in renal cell carcinoma: A systematic review. Crit Rev Oncol Hematol 161: 103331.

7. Song J, Yu Z, Dong B, Zhu M, Guo X, et al. (2021) Clinical significance of circulating tumour cells and Ki-67 in renal cell carcinoma. World J Surg Oncol 19: 156.

8. Cappelletti V, Verzoni E, Ratta R, Vismara M, Silvestri M, et al. (2020) Analysis of Single Circulating Tumor Cells in Renal Cell Carcinoma Reveals Phenotypic Heterogeneity and Genomic Alterations Related to Progression. Int J Mol Sci 21(4): 1475.

9. Kim TH, Kang YT, Cho YH, Kim JH, Jeong BC, et al. (2019) Detection of circulating tumour cells and their potential use as a biomarker for advanced renal cell carcinoma. Can Urol Assoc J 13(9): E285-e291.

10. Bai M, Zou B, Wang Z, Li P, Wang H, et al. (2018) Comparison of two detection systems for circulating tumor cells among patients with renal cell carcinoma. Int Urol Nephrol 50(10): 1801-1809.

11. Bluemke K, Bilkenroth U, Meye A, Fuessel S, Lautenschlaeger C, et al. (2009) Detection of circulating tumor cells in peripheral blood of patients with renal cell carcinoma correlates with prognosis. Cancer Epidemiol Biomarkers Prev 18(8): 2190-2194.

12. Nayak B, Panaiyadiyan S, Singh P, Karmakar S, Kaushal S, et al. (2021) Role of circulating tumor cells in patients with metastatic clear-cell renal cell carcinoma. Urol Oncol 39(2): 135.e139-135.e115.

13. Basso U, Facchinetti A, Rossi E, Maruzzo M, Conteduca V, et al. (2021) Prognostic Role of Circulating Tumor Cells in Metastatic Renal Cell Carcinoma: A Large, Multicenter, Prospective Trial. Oncologist.

14. Tsai WS, Hung TF, Chen JY, Huang SH, Chang YC (2021) Early Detection and Dynamic Changes of Circulating Tumor Cells in Transgenic NeuN Transgenic (NTTg) Mice with Spontaneous Breast Tumor Development. Cancers (Basel) 13(13): 3294.

15. Hassan S, Blick T, Thompson EW, Williams ED (2021) Diversity of Epithelial-Mesenchymal Phenotypes in Circulating Tumour Cells from Prostate Cancer Patient-Derived Xenograft Models. Cancers (Basel) 13(11): 2750.

16. Magbanua MJ, Das R, Polavarapu P, Park JW (2015) Approaches to isolation and molecular characterization of disseminated tumor cells. Oncotarget 6(31): 30715-30729.

17. Stevens M, Liu P, Niessink T, Mentink A, Abelmann L, et al. (2021) Optimal Halbach Configuration for Flow-through Immunomagnetic CTC
Enrichment. Diagnostics (Basel) 11(6): 1020

18. Allard WJ, Matera J, Miller MC, Repollet M, Connelly MC, et al. (2004) Tumor cells circulate in the peripheral blood of all major carcinomas but not in healthy subjects or patients with nonmalignant diseases. Clin Cancer Res 10(20): 6897-6904.

19. Zhou C (2012) Application of cell-search System to detect circulating tumor cells in patients with renal carcinoma. Doctoral dissertation, Peking Union Medical College, China.

20. Aceto N, Bardia A, Miyamoto DT, Donaldson MC, Wittner BS, et al. (2014) Circulating tumor cell clusters are oligoclonal precursors of breast cancer metastasis. Cell 158(5): 1110-1122.

21. Duda DG, Ancukiewicz M, Isakoff SJ, Krop IE, Jain RK (2014) Seeds and soil: unraveling the role of local tumor stroma in distant metastasis. J Natl Cancer Inst 106(8): dju187.

22. Alix Panabières C, Pantel K (2013) Circulating tumor cells: liquid biopsy of cancer. Clin Chem 59(1): 110-118.

23. Liu K, Gao R, Wu H, Wang Z, Han G (2021) Single-cell analysis reveals metastatic cell heterogeneity in clear cell renal cell carcinoma. J Cell Mol Med 25(9): 4260-4274.

24. Hanahan D, Weinberg RA (2011) Hallmarks of cancer: the next generation. Cell 144(5): 646-674.

25. Brown TC, Sankpal NV, Gillanders WE (2021) Functional Implications of the Dynamic Regulation of EpCAM during Epithelial-to-Mesenchymal Transition. Biomolecules 11(7): 956.

26. Paoletti C, Hayes DF (2016) Circulating Tumor Cells. Adv Exp Med Biol 882: 235-258.

27. Paterlini Brechot P, Benali NL (2007) Circulating tumor cells (CTC) detection: clinical impact and future directions. Cancer Lett 253(2): 180204.

28. Youn GJ, Chung WC (2017) Micrometastasis in Gastric Cancer. Korean J Gastroenterol 69(5): 270-277.

29. Hong B, Zu Y (2013) Detecting circulating tumor cells: current challenges and new trends. Theranostics 3(6): 377-394.

30. Hou JM, Krebs M, Ward T, Sloane R, Priest L, et al. (2011) Circulating tumor cells as a window on metastasis biology in lung cancer. Am J Pathol 178(3): 989-996.

31. Labelle M, Begum S, Hynes RO (2014) Platelets guide the formation of early metastatic niches. Proc Natl Acad Sci U S A 111(30): E3053-3061.

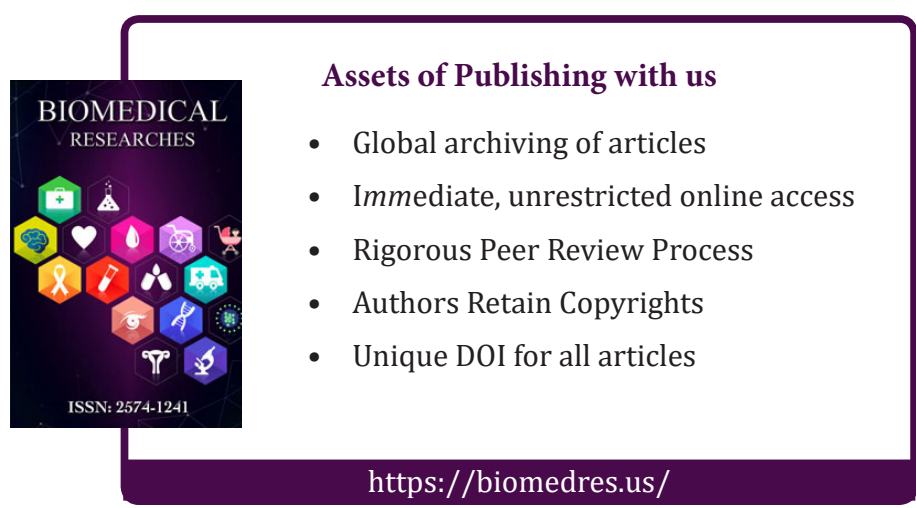

